\title{
“DESDE ARRIBA O DESDE ABAJO”: CONSTRUCCIONES Y ARTICULACIONES EN LA INVESTIGACIÓN SOBRE EDUCACIÓN INTERCULTURAL EN MÉXICO
}

\author{
"From Above or From Below": Constructions and Articulations of an Academic Research Field on \\ Intercultural Education in Mexico
}

Flor Marina Bermúdez-Urbina

\begin{abstract}
Resumen: En este artículo se realiza una aproximación a las comunidades epistémicas que conforman el subcampo de la educación intercultural en México y se reflexiona sobre los ámbitos y procesos que la articulan. Inicialmente, en el escrito se exponen las circunstancias que favorecen una transición de la investigación sobre la educación indígena hacia la educación intercultural y se explican las coyunturas que permiten su emergencia. En un segundo momento se describe el campo que articula las comunidades epistémicas diferenciadas por miradas "desde arriba y desde abajo". Se concluye con una reflexión sobre los desafios que enfrentan el estudio de las comunidades epistémicas y la investigación sobre educación intercultural en las coyunturas político-educativas actuales.
\end{abstract}

Palabras clave: interculturalidad, educación intercultural, comunidades epistémicas.

Abstract: This paper is an approach to the epistemic communities that make up the sub-field of intercultural education in Mexico and reflects on the scopes and processes which articulate them. Firstly, it presents the circumstances that favor the transition from the research on indigenous education towards intercultural education research and it explains the joints that allow its appearance. Secondly, it describes the field that articulates the epistemic communities contrasted by the looks "from above or from below". It concludes with a reflection on the challenges that the study of epistemic communities and the research on intercultural education faces in the current political-educational situations.

Keywords: interculturalism, intercultural education, epistemic communities.

Flor Marina Bermúdez Urbina, doctora en Pedagogía por la Facultad de Filosofía y Letras de la Universidad Nacional Autónoma de México. Académica del Consejo Nacional de Ciencia y Tecnología en el Centro de Investigaciones y Estudios Superiores en Antropología Social, unidad Pacifico-Sur, México. Temas de especialización: pedagogías de género e interculturales y educación superior intercultural. Correo electrónico: fmarinabermudez@hotmail.com.
Enviado a dictamen: 9 de marzo de 2015 Aprobación: 16 de abril de 2015.

Revisiones: 1. 


\section{Introducción ${ }^{1}$}

E propósito de este artículo es abonar a los debates existentes ${ }^{2}$ sobre la construcción de un campo de investigación social en educación intercultural en México. Su aporte se inscribe en una lectura analítica de este campo desde el concepto de "comunidad epistémica" desarrollado por Peter Hass (1992) y Luis Villoro (2006), un recurso utilizado para "el estudio del papel de las redes de investigadores en un país y de los expertos en un campo de estudio específico" (Maldonado, 2005a: 1).

La educación intercultural es definida como un concepto polisémico, comodín de los discursos políticos (Dietz y Mateos, 2011), ambiguo en su semántica (Medina, 2007), o como un concepto semiótico con capacidad de albergar todo tipo de ítems (González, 2013) y de acoger una amplia gama de enfoques teóricos, metodologías y reivindicaciones políticas de inclusión o diferencia inscritos en dimensiones teóricas, prácticas o programáticas diversas.

Su significado se articula con su origen. Maya Lorena Pérez Ruiz (2009: 256-257) considera que su emergencia en América Latina se inscribe en "una apuesta éticopolítica ante la imposición y fracaso de un modelo de nación sustentado en la homogeneización cultural y lingüística”, y Luis Enrique López (2009: 182-183) reconoce un proceso interactivo en el que "antropólogos y lingüistas en coordinación con intelectuales y dirigentes indígenas construyeron el imaginario de una sociedad multiétnica, pluricultural y multilingüe en un momento en que se construía un proyecto democrático en diversas partes del continente". López distingue la diversidad de apropiaciones, marcos teóricos y apuestas políticas que su incorporación adquiere en diversas partes de América Latina. Para Héctor Muñoz Cruz (2008), la educación intercultural bilingüe en México tiene dos pilares: el indigenismo oficial y el multiculturalismo de instituciones globales financieras, mientras que Gunther Dietz y Laura Mateos (2011) apuntan a la heterogeneidad de sus contextos de origen y a las diferentes adscripciones disciplinarias de sus actores. En su análisis de las "gramáticas discursivas de la interculturalidad" en un contexto globalizado, encuentran que la gramática de la diversidad en México mantiene una fuerte influencia ideológica nacionalista e indigenista y señalan, además, que abreva del multiculturalismo anglosajón y europeo, así como del interculturalismo latinoamericano.

Al considerar la polisemia del término y la complejidad inherente a su origen, en este artículo se busca profundizar en la diversidad de actores y reflexionar sobre los campos de tensiones, alianzas y divergencias que articulan a las comunidades epistémicas que debaten en torno a la educación intercultural en México.

Para el logro de este propósito, el texto se divide en tres partes: la primera se enfoca en describir la transición complementaria de la investigación sobre educación indígena hacia educación intercultural y las coyunturas que permiten su emergencia; en un segundo apartado se desarrolla una descripción y análisis del campo que articula a las comunidades epistémicas diferenciadas principalmente por miradas "desde arriba y desde abajo" ${ }^{3}$ de la educación intercultural, y se concluye con una reflexión sobre los desafíos que enfrenta la educación intercultural en las coyunturas político-educativas actuales.

\section{Conformación y desarrollo reciente en el campo}

La educación indígena en México y su abordaje como campo de conocimiento especializado cuenta con un largo trayecto ${ }^{4}$ (Bertely, 1998b; Ramírez, 2006). En su desarrollo ha profundizado en temas como: las políticas indigenistas o políticas de identidad (Dietz, 1999; Muñoz, 2001), la enseñanza de las lenguas originarias y las problemáticas inherentes al bilingüismo (Hamel y Muñoz, 1981; Muñoz, 2002; Hamel, 2000), los libros de texto (Corona, 2008), las apropiaciones étnicas de la escuela (Bertely, 1998a) y el papel de los profesores indígenas como profesionales (Jordá, 2003), participes de la aculturación (Caballero, 1989), intermediarios culturales (Vargas, 1994) o caciques culturales (Pineda, 1993). En la investigación desarrollada durante el último medio siglo destacan los trabajos de antropólogos como 
Julio de la Fuente y Nancy Modiano durante la década de los setenta, así como las sistematizaciones realizadas por intelectuales indígenas letrados durante los años ochenta y noventa (Hernández, 2000; Pérez Pérez, 2003). En estos mismos años, el campo comenzó a crecer con la incorporación de historiadores, lingüistas, pedagogos y sociólogos que profundizaron en la comprensión de las problemáticas inherentes a la educación indígena en contextos locales, regionales o nacionales.

A partir de los años noventa e inicios de este siglo, dos coyunturas históricas marcaron las dimensiones actuales de la educación intercultural. La primera se inscribe en la emergencia política que detonó el levantamiento zapatista en la Selva de Chiapas a partir del año 1994 (Dietz, 2009) y que favoreció una rearticulación de actores y organizaciones indígenas que impulsaron un cuerpo de demandas enfocadas en la autonomía indígena y en el cumplimiento del derecho a una "educación propia". ${ }^{5}$ El movimiento intelectual cercano al neozapatismo promovió, a su vez, un reposicionamiento de actores académicos que asumieron un compromiso político con el movimiento y el ejercicio de sus demandas políticas y educativas (Leyva y Speed, 2008).

La segunda coyuntura de cambio para la educación dirigida a los pueblos originarios se inscribe en la institucionalización y oficialización de la educación intercultural a partir de la creación de la Coordinación General de Educación Intercultural Bilingüe (CGEIB) en el año 2001, ${ }^{6}$ instancia con atribuciones de planeación educativa que asumió la administración de la educación intercultural en el contexto nacional (Bertely, 2013).

Esta instancia gubernamental se estableció como "administradora del conflicto" derivado de las múltiples y variadas demandas de reconocimiento a la diversidad que emergían aceleradamente en el país en la era del multiculturalismo neoliberal (Hale y Millamán, 2007). La apuesta de una institucionalización de la educación intercultural fortaleció de manera relativa las posturas de la CGEIB y se expresó en las atribuciones que le fueron conferidas y que abarcan: la definición de un modelo educativo intercultural, la formulación de programas de formación para docentes indígenas y no indígenas (Baronnet, 2010), su papel "regulador" en la creación de nuevos subsistemas educativos, como los bachilleratos y universidades interculturales (Dietz y Mateos, 2011), así como su participación decisiva en la elaboración de materiales didácticos con enfoque intercultural, su aval en publicaciones científicas y el financiamiento de investigación especializada en el tema.

La institucionalización de la educación intercultural como proyecto político-educativo del Estado ha registrado variados campos de incidencia y ha favorecido la formación de grupos de investigación especializados en la temática, que emergieron de forma significativa a mediados del año 2000 con la creación de cuerpos académicos en universidades públicas estatales, la consolidación de grupos de investigación en centros públicos y privados ${ }^{7}$ nacionales y la creación de programas de formación, como diplomados, licenciaturas y posgrados. Es importante mencionar que estos grupos de investigación e investigadores interesados en la educación intercultural se articulan con trabajos y nichos de producción y debate académico existentes desde la década de los noventa. ${ }^{8}$

Entre algunos actores académicos se registra un desplazamiento de una investigación anteriormente centrada en la educación indígena, las lenguas indígenas o el bilingüismo escolar, hacia la educación intercultural. Puede suponerse que esta evolución estuvo fuertemente atravesada por los procesos de "legitimización institucional" del concepto de interculturalidad. ${ }^{9}$

Paralasinstancias oficiales, desplazar discursivamente la educación indígena -bilingüista, castellanizadora e integradora - hacia la noción de educación intercultural "para todos" (Schmelkes, 2004) ${ }^{10}$ permitió que, en una coyuntura política de importantes reclamos de reconocimiento a la diferencia y al acceso a una educación culturalmente pertinente, la educación intercultural operara como concepto innovador y de amplios significados y pudiera ser utilizada como recurso "esperanzador" en un contexto de profundos cuestionamientos sobre las condiciones de desigualdad, segregación y racismo institucional que han permeado a la educación indígena. 
Es importante distinguir - $-\mathrm{y}$ en ello estriba en parte la relevancia de este escrito- los procesos de negociación e interacción entre la CGEIB, los nuevos movimientos sociales, las luchas indígenas y las comunidades epistémicas, procesos que no han sido lineales, sino que han favorecido variadas apropiaciones y resignificaciones del término en los planos discursivos, programáticos y pragmáticos, y que han generado una variedad y profundidad en el campo de estudio de la educación intercultural en el país.

\section{Comunidades epistémicas en la educación intercultural}

Una comunidad epistémica es una red de profesionales con reconocida experiencia y competencia de un dominio en particular y con autoridad para producir conocimiento relevante para la política. Los miembros de una comunidad epistémica comparten entendimientos intersubjetivos y patrones de razonamiento, y desarrollan un proyecto político sobre la base de valores compartidos, además de tener un compromiso con la aplicación y la producción de conocimiento ${ }^{11}$ (Haas, 1992: 3-4).

Para Haas (1992: 4), las comunidades epistémicas no son lo mismo que las comunidades científicas, ya que las primeras no se refieren exclusivamente a la producción de conocimiento, sino que más bien se trata de fuertes actores en lo nacional que participan de la toma de decisiones al proporcionar información valiosa para la formulación de políticas. Adicionalmente a esta definición, me interesa proponer un ámbito de imbricación mayor de las comunidades epistémicas al reconocer su potencial no solamente en la formulación de políticas de incidencia gubernamental, sino también en su articulación con actores sociales de "base" sobre los que influyen de distintas maneras al favorecer la circulación de conocimientos respecto a los enfoques o discusiones transcontinentales de la interculturalidad, y al proporcionarles recursos que les permiten impulsar desde posiciones periféricas agendas políticas con reivindicaciones mayores a las concedidas por el Estado.
Es relevante señalar que Villoro (2006: 147) amplía la concepción de comunidad epistémica al considerar que "todo sujeto forma parte de una comunidad epistémica determinada y constituida por todos los sujetos epistémicos posibles que tengan acceso a las mismas razones". Para Villoro (2006) no existe una membresía exclusiva de la comunidad científica hacia las comunidades epistémicas porque estas son algo más que los grupos de interés. Reconoce además un potencial del sujeto -incluyendo al sujeto social no academizado - en la construcción de nuevos conocimientos, paradigmas y el consenso en sistemas de creencias y conocimientos, debido a que todo saber está condicionado socialmente.

En México, la comunidad científica ha dado paso a la existencia de variadas comunidades epistémicas; sin embargo, cada una de ellas ejerce niveles distintos de influencia en la formulación de políticas públicas y programas de gobierno. Maldonado (2005b) señala que el estudio de las comunidades epistémicas en México apenas inicia y se configura como un campo emergente para explicar las imbricaciones entre investigación e incidencia pública. En este artículo no busco analizar concretamente la influencia de las redes de expertos en la instauración de las políticas educativas interculturales. Me propongo un análisis más modesto de caracterización de los actores y grupos de expertos o especialistas en el campo de la educación intercultural y su influencia en la definición y distinción analítica de paradigmas interculturales "desde abajo y desde arriba".

\section{El subcampo de la educación intercultural en México}

La educación intercultural "desde arriba y desde abajo" coexiste en lo que Pierre Bourdieu llamaría, dentro del campo de la educación, el subcampo de la educación intercultural. El campo y sus subcampos son conceptualizados como una red de relaciones estructuradas y objetivas entre diversas posiciones y actores en la que se desarrollan disputas y luchas por la hegemonía. 
Los subcampos deben ser entendidos como espacios de conexión entre redes de intercambio que comparten saberes y experiencias que, si bien disputan posiciones de poder y representación, también tejen alianzas estratégicas para el logro de objetivos comunes.

Recientemente, en el subcampo de la educación intercultural se articulan académicos formados en distintas áreas disciplinares, aunque predomina la presencia de antropólogos, sociólogos, lingüistas y especialistas en la pedagogía o ciencias de la educación. En menor proporción, también académicos especialistas en comunicación, arte, epistemología de la ciencia o literatura desarrollan investigaciones sobre el tema. ${ }^{12}$

El predominio de los antropólogos tiene su origen en la influencia de la antropología mexicana en la formulación de las políticas indigenistas y en la transición "inacabada" de la educación indígena a la educación intercultural calificada como neoindigenista (Cerda, 2007), que atribuye un énfasis esencialista a las culturas indígenas y que tiene como objeto de atención procesos étnicos e identitarios de los pueblos originarios. ${ }^{13}$ Otra vertiente llamada crítica se interesa por la explicación del conflicto de poder, representación y recursos en juego entre una mayoría mestiza y una clase indígena dominada. Se busca explicar al otro en el "inter" de la cultura indígena — asimilada como igual a la identidad étnica-, que se materializa en un entorno de múltiples desventajas frente a la cultura mestiza mayoritaria. La socioantropología encuentra en la educación intercultural un asidero para continuar explicando procesos identitarios, étnicos, políticos, de poder y de conocimiento indígena en contextos escolares y comunitarios (Rockwell y González, 2012).

Sobre estas posturas que cuentan con una fuerte influencia en la agenda de investigación, se desarrollan también reflexiones sobre la comunicación intercultural (Corona, 2008; Corona y Pérez, 2009), la epistemología de la ciencia o ciencia intercultural (Olivé, 2009; Cuevas, 2013; Lazos y García, 2011), la filosofía y epistemologías indígenas, el géneroy la interculturalidad (Bermúdez, 2013), y sobre gramáticas interculturales y decolonialidad (Medina, 2009). Cabe mencionar que resultan casi inexistentes estudios sobre afromexicanos e investigaciones diacrónicas de la educación indígena e intercultural (Bertely, Dietz y Tepepa, 2015).

La distribución por instituciones de los agentes/ actores de la investigación intercultural también muestra una composición interesante; paulatinamente se fortalece e incrementa la producción de grupos de investigación ubicados fuera de la capital del país y se robustece la producción académica en universidades estatales (Bertely, Dietz y Tepepa, 2015). Sin embargo, la gran mayoría de las universidades interculturales ocupa posiciones periféricas en la distribución de recursos, en la formulación de políticas públicas y en la producción de conocimiento sobre educación intercultural. ${ }^{14}$

Destaca en la composición de este subcampo la presencia de organismos internacionales de diverso perfil que financian y orientan la implementación de políticas y programas sobre educación intercultural. Por una parte, destaca la presencia de la Organización de las Naciones Unidas para la Educación, la Ciencia y la Cultura (UNESCO) y del Banco Mundial, que han orientando las concepciones sobre cultura y diversidad cultural que asume la CGEIB en México (CGEIB, 2014). De igual forma, el Instituto Internacional para la Educación Superior en América Latina (IESALCUNESCO) ha incidido en la formulación de políticas relacionadas con la educación superior intercultural, vinculando a expertos nacionales y sus instituciones con este organismo. Organizaciones como el Grupo Internacional de Trabajo sobre Asuntos Indígenas (IWGIA) y la Fundación Ford han financiado propuestas impulsadas por organizaciones indígenas de base que buscan consolidar su autonomía frente a las instancias oficiales e imprimen una agenda a las organizaciones que financian..$^{15}$

En el ámbito del financiamiento de la investigación ${ }^{16}$ se registran políticas sectorizadas a través de dependencias como el Consejo Nacional de Ciencia y Tecnología (CONACyT), instancia que en el año 2012 emitió una convocatoria en la que destinaba un fondo especial de investigación para la educación indígena e intercultural. ${ }^{17}$ Con este primer fondo específico para el área, el CONACyT financió trece proyectos, de los cuales diez fueron para universidades o centros de 
investigación ubicados en entidades de provincia. Esta convocatoria ha tenido como principales limitantes que los fondos constituyen una bolsa de recursos modesta - cuatro millones de pesos aproximadamente- y que hasta ahora no presenta continuidad para las investigaciones financiadas en este primer esfuerzo y las nuevas propuestas que pudieran existir.

Uno de los ámbitos de mayor peso en la articulación del subcampo se sitúa en la producción académica especializada en el tema. En México, un referente obligado de consulta son los estados de conocimiento promovidos por el Consejo Mexicano de Investigación Educativa (COMIE) en los últimos veinte años. La elaboración de estos documentos ha sido coordinada por especialistas destacados (Delgadoet al., 1995; Bertely, 2003; Bertely, Dietz y Tepepa, 2015).

En su análisis sobre la producción académica en la última década (2003-2013), Bertely, Dietz y Tepepa (2015) apuntan la existencia de un crecimiento en la producción — 884 referencias, siendo las principales fuentes libros por capítulo, libros por autor y artículos de revistas - Registran, por una parte, la existencia de investigación sobre educación intercultural con escasa incidencia en la práctica y, por otra, la emergencia de actores etnopolíticos que luchan por una nueva educación articulados con la academia. Bertely, Dietz y Tepepa (2015) ubican la agencia de investigadores que dialogan, asesoran y colaboran con instancias oficiales de gobierno, como la CGEIB y el Instituto Nacional de Lenguas Indígenas (INALI), en estudios o colaboraciones coyunturales. También señalan la actividad de investigadores sin vínculos gubernamentales o de base ocupados en la producción de textos científicos sobre interculturalidad que implementan metodologías convencionales, mientras que otros persiguen la articulación con organizaciones de base para incidir en sus agendas y otorgar recursos para su formación. Otros más — una minoría- poseen habilidades para transitar en el diálogo y la negociación entre instancias gubernamentales, sectores de base y la academia, lo que muestra una diversificación de actores y de prácticas de investigación, y su articulación con actores estratégicos.
Se observa además un incremento de los espacios de intercambio y redes de colaboración. Bertely, Dietz y Tepepa (2015) destacan los congresos nacionales de Investigación Educativa, el Seminario Escuela, Indígenas y Etnicidad - coordinado por CIESAS, en el que participan siete sedes remotas-, el Foro Latinoamericano de Educación Intercultural, Migración y Vida Escolar, los encuentros de la RedFEIAL, así como los congresos nacionales de Educación Indígena e Intercultural o el Congreso Latinoamericano de Educación Intercultural, entre otros. En estos espacios, académicos, funcionarios, integrantes de organizaciones no gubernamentales y grupos implicados en la formación de políticas públicas comparten y socializan debates que hacen aflorar diferencias entre enfoques "desde arriba, desde abajo o sin posicionamiento explícito alguno", que atraviesan la práctica profesional de los investigadores y establecen, como nuevo dilema ético-político, para quiénes y para qué realizar investigación intercultural, así como el papel de los indígenas en la autoría y planeación de sus procesos educativos. En estos espacios de articulación colectiva se busca también reflexionar de manera crítica en torno al compromiso de los investigadores/ académicos (Baronnet, Mora y Stahler, 2011) con los sujetos que participan de la educación intercultural y su relación con las instancias oficiales en contextos de desequilibrio de poder e incidencia.

\section{Desde arriba y desde abajo, el locus de enunciación de la educación intercultural}

Desde el año 2003, en el prólogo redactado para el estado de conocimiento del COMIE, María Bertely (2003) identificaba el papel "incómodo" que la investigación sobre educación, derechos sociales y equidad parecía generar entre los otros campos de la investigación educativa que desarrollaban indagaciones paralelas. En su escrito, Bertely (2003: XXVII) identificaba los "locus de enunciación" - horizontes de inteligibilidad, perspectivas y posiciones- a partir de los cuales se habían producido materiales con enfoque indígena o intercultural. Bertely (2003: 19) realizó una clara 
distinción entre la enunciación que realizaban los planificadores de políticas interculturales, frente a las perspectivas de los actores en regiones, pueblos y grupos étnicos específicos, siendo estos últimos, en algunos casos, menos enunciados y recuperados en la producción académica — autoría nativa-

Tres años después, Luis Enrique López (2006) publicó un texto en el que aludía a los dos principales locus de enunciación en la educación intercultural, "desde arriba y desde abajo", en su revisión sobre las luchas más importantes de los pueblos indígenas en América Latina. En el documento resaltó el importante peso que tienen para las organizaciones indígenas la cobertura, la extensión de la educación intercultural bilingüe, la transformación del currículum oficial, la revitalización de las lenguas indígenas y el interculturalizar a las mayorías mestizas. De estos procesos, rescató el importante posicionamiento de los actores de la educación intercultural bilingüe en la formación de una conciencia política crítica y su papel como agentes activos en la construcción y desarrollo de los proyectos educativos.

En este sentido, "la educación intercultural desde abajo" fue definida por Erica González Apodaca y Angélica Rojas Cortés (2013: 275) como proyectos educativos "políticamente posicionados y cercanos a la investigación colaborativa, el activismo social y la reivindicación étnica". Las autoras muestran que la educación intercultural en contextos específicos donde existen entramados étnicos históricos y actores que impulsan procesos de etnogénesis y reinvención cultural, más que referir solamente a una concepción superficial de la diversidad, persigue en muchos casos fines etnopolíticos y plantea la reivindicación de derechos colectivos y autonomía, a la vez que se aspira a recuperar demandas arraigadas en procesos históricos de reflexión colectiva, grupal o individual, y de análisis de la realidad de quienes las formulan (González y Rojas, 2013: 276-277).

La interculturalidad desde abajo resemantizada por actores sociales políticamente posicionados, tanto de la academia como de sectores de base, puede constituirse en un: "camino de apropiación y ejercicio de los sustentos político-jurídicos, filosóficos, pedagógicos y éticos de una propuesta educativa-política". La investigación desde abajo pone a discusión temas como la autoría -investigación dialógica, interactoral u horizontal-y recurre a metodologías colaborativas entre académicos y actores locales (González y Rojas, 2013: 276-277). Las comunidades epistémicas que participan de este campo plantean temas como el diálogo de saberes, saberessaberes, saberes-haceres y saberes-poderes como recursos potencialmente descolonizadores del sistema educativo mexicano (Dietz y Mateos, 2011: 171) y se dirigen hacia una construcción epistémica de la interculturalidad.

Este tipo de investigación se interesa por el estudio de propuestas o proyectos que tienen como propósito en algunos casos la construcción de la autonomía - expresada explícita o implícitamente en diferentes ámbitos y niveles - y que se definen como alternativos a las propuestas educativas existentes en la educación oficial. Los investigadores que configuran este campo de investigación se interesan por estudiar e intervenir en el análisis de los procesos etnogenéticos, la recuperación de conocimientos culturales propios y las propuestas de educación con incidencia política, a la vez que formulan el desarrollo de una conciencia crítica en el debate sobre temas centrales de los pueblos indígenas, como el territorio, la lengua y los derechos.

La investigación surge a partir de la demanda de los pueblos originarios, que reclaman la asesoría de sus intelectuales letrados y de académicos comprometidos con la defensa de los pueblos originarios. La educación intercultural "desde abajo" se asemeja a una hibridación resultante de un proceso de colaboración o intermediación que ha permitido la formación de cuadros indígenas empoderados con capacidad para reflexionar acerca de su realidad y gestionar recursos para su propio desarrollo, con capacidades para la formulación de apuestas teóricas desde epistemologías propias. Los desafíos que enfrentan son el escaso financiamiento, la tendencia a la esencialización y a la reiteración de estereotipos idealizados sobre los pueblos indígenas - cercanos a la naturaleza, democráticos y comunitaristas-, y la articulación finalmente jerárquica entre los actores locales y los investigadores, lo que deviene en variadas dudas sobre la producción 
colectiva cuando las motivaciones de los actores - bases e investigadores - son diferentes.

Es importante hacer notar que una postura "desde abajo" no debe ser entendida como una categoría cerrada ni descriptiva, sino que debe ser contextualizada de acuerdo con la heterogeneidad y tensiones que articulan el campo. "Desde abajo" es una posición que permite resaltar las connotaciones de poder en las que se producen conocimientos que han sido hegemonizados a partir de ciertos significados.

Por su parte, la existencia de una "educación intercultural desde arriba" deviene de los procesos de institucionalización de la interculturalidad en el contexto mexicano (Cerda, 2007; Fuentes, 2010), y se explica también a partir de una relación de poder claramente definida en la que el Estado mexicano concede nuevos espacios educativos en una actitud de pluralismo condescendiente (Dietz y Mateos, 2011). Si bien los procesos de apropiación de la interculturalidad desde arriba atienden en parte demandas de reconocimiento "jurídico" y "de representación", han vaciado su potencial de transformación en el campo de los derechos políticos y se han refuncionalizado desde una perspectiva reduccionista de sus implicaciones (Cerda, 2007). Una característica de esta interculturalidad desde arriba estriba en su "centralismo", que se expresa en la formulación de un modelo nacional para la educación intercultural y en una coordinación general (CGEIB) encargada de gestionar, administrar y "dosificar" la interculturalidad en sectores estratégicos.

El reclamo más importante a este tipo de interculturalidad es que se anula al indígena como un sujeto de derecho (Medina, 2009), lo que se expresa en la negación de que los pueblos indígenas puedan crear y coordinar sus propias instituciones educativas vinculadas al sistema oficial (Cerda, 2007: 101). Este modelo convierte al sujeto indígena en un "beneficiario" de programas que promueven sutilmente su integración nacional o global y lo perfilan como un agente que debe conocer lo que tiene - recursos materiales y simbólicos - para desarrollar competencias sociales que lo hagan eficiente en un mundo globalizado y competitivo (Hale, 2002). La interculturalidad desde arriba tiende a reproducir construcciones del indígena como un sujeto ahistórico con una cultura folklorizada y esencializada, como un sujeto que debe tener a la vez una fuerte identidad nacional y capacidad de negociar su diversidad cultural en un entorno nacional desigual.

Los estrechos márgenes para la participación de los pueblos originarios en la formulación y apropiación endógena de la educación intercultural institucionalizada también forman parte de la experiencia de sectores académicos que participan con las instancias gubernamentales encargadas de su administración.

Los agentes encargados de gestionar la interculturalidad oficial permiten que actores y grupos académicos "selectos" desarrollen variados marcos de interacción, participación y colaboración en la formulación de propuestas y proyectos interculturales — evaluación de proyectos, paneles de especialistas-; sin embargo, devienen variadas reflexiones respecto a los marcos de participación establecidos y su capacidad de transformación al sistema educativo y el currículum nacional ${ }^{18}$ (Dietz, 2014).

La participación de actores académicos en los ámbitos oficiales ha tenido gran peso en la formulación y legitimación de propuestas pedagógicas diversas, pero ha incidido poco en la formulación de la política y en la transformación de concepciones estereotipadas, nacionalistas, y en algunos casos racistas, en los materiales educativos. El talón de Aquiles de la investigación académica en el campo de la educación intercultural oficial apunta a su escasa o débil incidencia en la mejora de las condiciones en las que se imparte la educación para los pueblos originarios y su capacidad de afrontar con estrategias exitosas la "marginalidad sistémica"19 que enfrenta la educación intercultural en su conjunto.

\section{Reflexiones finales sobre los desafíos de las comunidades epistémicas de educación intercultural en México}

Hasta aquí hemos planteado una ampliación del modelo de comunidad epistémica propuesto por Haas (1992) a 
partir del hecho de que las comunidades epistémicas en la educación intercultural en México pueden ser exploradas -desde esta propuesta inicial para su abordaje - a partir de su articulación en tres planos: la incidencia en políticas educativas gubernamentales, su preponderancia en los temas prioritarios de producción en investigación y escritos, y su incidencia sobre actores de base.

La concepción de una "comunidad epistémica dialógica", con múltiples capacidades de articulación entre sí, imprime a los investigadores del campo importantes desafíos. Uno de ellos es reconocer y dialogar con posiciones periféricas colocadas "en medio", ni arriba ni abajo, que también buscan un espacio de reconocimiento e interlocución que les permita producir conocimiento de avanzada e interactuar con los agentes hegemónicos que debaten en el campo. El arriba y el abajo, como locus de enunciación con un entramado complejo de actores, agendas y demandas, poseen también imbricaciones que los articulan, y de ahí deriva en parte la complejidad del campo. Es necesario llevar la investigación sobre la educación intercultural a espacios multidisciplinares y complementarios en los que la producción periférica sea reconocida en su potencial heurístico y como aporte al conocimiento de las realidades complejas que dan sentido a la educación intercultural.

Al recuperar la propuesta de Luis Enrique López (2006) respecto a una interculturalidad desde arriba o desde abajo, no buscamos ceñir la configuración del campo a una postura dicotómica, sino más bien intentar ampliar el debate respecto a la necesidad de encontrar puntos de traspasamiento y conexión, intersticios donde emerjan propuestas que no se ciñan a estas dos posturas, pero que construyan proyectos educativos interculturales en redes interactorales diversas.

A partir de los tres ámbitos de incidencia de las comunidades epistémicas y de su necesario carácter dialógico arriba mencionado, podemos perfilar algunos desafíos para su consolidación en lo epistémico, lo teórico y lo metodológico. De la recuperación de estas propuestas deriva, en parte, la capacidad de incrementar su incidencia sobre la política gubernamental y su consolidación con sectores de base con los que buscan producir procesos de transformación de largo alcance.

El primer desafío se inscribe en lo que Dietz llama terminar con una "marginalidad sistémica" de la interculturalidad; de manera procesual, se trata de la marginación que atraviesa un velado racismo institucional y epistémico (Dietz, 2011). Esta posición de la educación intercultural como vocablo central en el discurso, pero periférico en su transversalización institucional, revela juegos complejos de poder y exclusión que se ejercen desde instituciones educativas, políticas y económicas, y que reproducen desde su posición hegemónica la exclusión histórica hacia los pueblos originarios.

En este sentido, la preponderancia de las agendas determinadas por la interculturalidad "desde arriba" ha favorecido la formulación de campos de investigación e intervención interculturales que parecieron haber incidido poco en las estructuras de poder y desigualdad existentes, y de ahí deriva un interés mayor por la pedagogización de prácticas culturales que en su formulación frecuentemente promueven posturas preservacionistas de las lenguas indígenas y la cultura, lo que mengua su potencial político y emancipatorio, ciñendo con ello el margen de exigibilidad de sus derechos. La esencialización de la cultura indígena - para bien o para mal- ${ }^{20}$ reproduce un racismo cultural velado que entiende la desigualdad en términos de diferencias culturales, que manipula discursos de inferioridad y que acentúa la dicotomía entre pueblos indígenas y la mayoría mestiza (Hale, 2004). Estos enfoques arruinan la capacidad de reinvención y resemantización de la educación intercultural en los múltiples contextos de diversidad cultural.

Los retos son evidentes y son motivo de discusión entre los grupos académicos que debaten en torno a este campo. Los especialistas en la materia reconocen importantes desafíos para los estudios sobre educación intercultural e identifican la necesidad de partir de la categoría de diversidad (Dietz y Mateos, 2011) para llegar al anhelado "inter", y desde esta posición epistémica debatir temas transversales como son: los géneros -y sus pluralidades-, las identidades trans ${ }^{21}$ las nuevas 
construcciones juveniles indígenas resemantizadas en su indianidad, las identidades indígenas periféricas articuladas a las migraciones materiales y simbólicas que han generado renovadas apropiaciones del territorio, la pertenencia y lo ritual, los renovados usos de las lenguas originarias en contextos multiculturales y su proyección como recurso variado.

Finalmente, un desafío importante se ubica en la necesaria renovación de la educación intercultural oficial, que motiva la necesidad de ampliar las construcciones conceptuales hasta ahora existentes de la interculturalidad y de formular conocimientos de frontera o contrahegemónicos (Lins y Escobar, 2009) que incidan en quienes toman decisiones para que renueven sus concepciones sobre la cultura, la diversidad y la pluralidad. Podemos pensar en una investigación en educación intercultural que muestre su potencial heurístico en la comprensión y, por qué no, en la transformación de una sociedad mexicana fragmentada por desigualdades históricas y que requiere de recursos renovados para enfrentar las diversas crisis estructurales por las que atraviesa.

\section{Notas}

${ }^{1}$ Las reflexiones aquí expuestas han germinado y se han alimentado en el marco de las actividades que desarrollamos colectivamente en el proyecto de investigación: "Políticas de interculturalidad en la educación superior en Oaxaca, proyectos educativos etno-políticos en la construcción de un campo social". El proyecto es coordinado por la Dra. Erica González Apodaca (CIESAS-Pacífico Sur) y participan los doctores Angélica Rojas Cortes, Arturo Tapia Guerrero -investigadores del programa Cátedras CONACyT para jóvenes investigadores (2014) comisionados al CIESAS unidad Pacifico Sur- y la doctora Olga Grijalva, profesora-investigadora del ICE-UABJO.

${ }^{2}$ Los antecedentes previos a esta discusión se encuentran en los textos: Bertely (2003), Bertely y González Apodaca (2004), Schmelkes (2004), López (2006), Pérez Ruiz (2009), Dietz y Mateos (2011), Rockwell y González Apoca (2012) y Bertely (2013).
${ }^{3}$ En el año 2006 Luis Enrique López publicó el artículo titulado: "Desde arriba y desde abajo: visiones contrapuestas de la educación intercultural bilingüe en América Latina". En este texto alude a las dos visiones predominantes de la educación intercultural en el continente americano, describiéndolas por separado.

${ }^{4}$ No es propósito de este escrito hacer una revisión - estado de conocimiento- de la educación indígena en México, solamente se mencionan superficialmente algunos de sus temas recurrentes y a autores que cuentan con textos sobre los temas apuntados. Para una revisión detallada sobre los estudios en antropología de la educación en México, revisar el texto de Rockwell y González (2012).

${ }^{5} \mathrm{Si}$ bien el movimiento neozapatista mexicano es uno de los referentes más significativos, es importante no perder de vista que décadas atrás otros actores y organizaciones indígenas habían impulsado agendas que reivindicaban diversos tipos de derechos para los pueblos originarios, aun cuando su discurso no era primordialmente etnopolítico.

${ }^{6}$ Para una descripción detallada de este proceso, ver los textos de Fuentes (2010) y Cerda (2007).

${ }^{7}$ Destacan la Universidad Iberoamericana y el ITESO. Es importante aclarar que la investigación en estas instituciones parte principalmente del interés individual de ciertos investigadores y estudiantes; no es posible hablar de grupos de investigación en el sentido en que se expresa en otras instituciones.

${ }^{8}$ Destacan el SEIE (Seminario Escuela, Indígenas y Etnicidad), impulsado por el CIESAS, y los seminarios de etnografía de la educación desarrollados en el DIE del CINVESTAV, entre otros.

${ }^{9}$ Si bien las revisiones realizadas sobre la producción reciente en el campo (Bertely, Dietz y Tepepa, 2013) parecieran apuntar hacia un predominio de la investigación en educación intercultural, es importante señalar que la investigación sobre educación indígena sigue desarrollándose con cierta independencia.

${ }^{10}$ Cabe señalar que este desplazamiento fue más sutil de lo que pudiera pensarse, ya que a la anterior denominación de "educación bilingüe bicultural" se le añadió la palabra "intercultural", por lo que desde inicios 
de 2000 recibe el nombre de "educación intercultural bilingüe" (EIB). Este desplazamiento se apoyó en la legitimidad lograda por la EIB en América Latina (López y Küper, 1999). Lo importante de este proceso es que, a partir de la acepción "educación intercultural para todos", se señala que la EIB es para todos, indígenas y no indígenas, aunque en las propuestas programáticas y prácticas implementadas por la CGEIB son mínimas las acciones que consideran la interculturalidad como acción transversal para la mayoría mestiza nacional.

${ }^{11}$ Traducción propia.

${ }^{12}$ En contraste, Teresa Aguado (2004) y Vallejo et al. (2005), en sus estudios sobre la producción académica en educación intercultural en España, identifican una composición multidisciplinar con prevalencia de especialistas en los campos de la psicología, la pedagogía y la sociología interesados en el estudio de la diversidad.

${ }^{13}$ LaCGEIBdestinaunaparteimportantedesuproducción a videos o libros de divulgación de materiales en lenguas indígenas dirigidos a los grupos étnicos del país. En menor medida se registra la existencia de materiales pedagógicos que promuevan interculturalizar a la mayoría mestiza nacional. Consultar sitio web de la CGEIB: http://eib.sep.gob.mx/cgeib/biblioteca-virtual/ editar-para-la-diversidad/.

${ }^{14}$ Comunicación personal con Gunther Dietz en el taller sobre universidades interculturales, en el marco de la reunión de la RedFEIAL, celebrada en Oaxaca de Juárez en febrero de 2015.

15 En Latinoamérica, instituciones de cooperación internacional como GTZ - ahora GIZ - han financiado e impulsado enfoques interculturales descolonizadores que han incidido profundamente en la formación de líderes indígenas y en la formulación de currículos interculturales. Asimismo, desde las organizaciones de base han impulsado agendas con fuerte incidencia en la política educativa de la nación bolivariana (López y Küper, 1999).

${ }^{16}$ Habría que mencionar también al PRODEP, Programa para el Desarrollo Profesional Docente de la Secretaría de Educación Pública. Aunque no se encontraron datos disponibles de los montos históricos financiados a cuerpos académicos por área de conocimiento, esta dependencia apoya de manera significativa a grupos de investigación en formación o consolidados, así como a profesores recién incorporados a las instituciones o que han obtenido un grado académico al recibir una beca dentro del programa.

${ }^{17}$ Información de los proyectos aprobados disponible en: http://www.conacyt.mx/index.php/el-conacyt/ convocatorias-y-resultados-conacyt/convocatoriasfondos-sectoriales-constituidos/convocatoria-sepconacyt/sep-cgeibconacyt/padron-cgeib.

${ }^{18}$ Este análisis se retoma de la videoconferencia impartida por Gunther Dietz el 5 de noviembre de 2014, en las instalaciones del CIESAS, unidad Pacifico Sur, en Oaxaca de Juárez, México.

${ }^{19}$ Comunicación personal con Gunther Dietz en el taller sobre universidades interculturales, en el marco de la reunión de la RedFEIAL celebrada en Oaxaca de Juárez, en febrero de 2015.

${ }^{20}$ La cultura indígena tiende a ser esencializada en dos sentidos: como "un legado valioso" herencia de los pueblos fundacionales del México antiguo, exaltando su valor en el pasado - monumentos, literatura-, o bien, como el indígena actual, salvaje, irracional, atrasado, sucio, pobre y causa de los atrasos del país.

${ }^{21}$ Lo trans no es un inter (entre territorios), sino un "más allá de". Con lo trans se genera otro territorio. No se pasa una frontera, sino que se transgrede. Una frontera se cruza o se atraviesa, se penetra, tal vez se transforma o se supera. Los contenidos de esos espacios, de esos cuerpos que se atraviesan, quedan transgredidos, afectados. Lo trans aplaza o desplaza. Al decir lo trans, se cambia la perspectiva del sujeto y su relación con el objeto. Lo trans genera un campo de existencia de algo complejo (Belausteguigoitia, 2015).

\section{Referencias bibliográficas}

Aguado Odina, Teresa (2004), “Investigación en educación intercultural”. En Educatio, núm. 22, pp. 39-57.

Baronnet, Bruno (2010), "De cara al currículum nacional. Las escuelas normales indígenas en las políticas de formación docente en México". En Saúl Velasco Cruzy 
Alexandra Jablonska (coords.), Construcción de políticas educativas interculturales en México: debates, tendencias, problemas, desafíos. México: UPN, pp. 245-272.

Baronnet, Bruno, Mariana Mora Bayo y Richard StahlerSholk (2011), Luchas muy otras. Zapatismo y autonomía en las comunidades indígenas de Chiapas. México: UAM Xochimilco, CIESAS, UNACH.

Belausteguigoitia, Marisa (2015), Lo trans: transcripción. S.l.: Instituto de Estudios Latinoamericanos, Universidad de Berlín. Disponible en: http://www.lai. fu-berlin.de/es/e-learning/projekte/frauen_konzepte/ projektseiten/konzeptebereich/be_trans/transcrip/ index.html [consultado el 6 de febrero de 2015].

Bermúdez Urbina, Flor Marina (2013), “Como ingenieras cuidamos mejor las plantas'. La situación de género de mujeres universitarias indígenas mam, en la Sierra de Chiapas, México". En Zona Franca, Revista del Centro de Estudios Interdisciplinario sobre Mujeres, Universidad Rosario Argentina, año XXI, núm. 22, pp. 65-74.

Bertely Busquets, María (1998a), "Historia social de la escolarización yuso del castellano escrito en un pueblo zapoteco migrante". Tesis de doctorado en Educación, Universidad Autónoma de Aguascalientes, México.

Bertely Busquets, María (1998b), "Educación indígena del siglo XX en México". En Pablo Latapí (coord.), Un siglo de educación en México. México: FCE, CNCA, pp.74-110.

Bertely Busquets, María (coord.) (2003), Educación, derechos sociales y equidad. México: COMIE.

Bertely Busquets, María y Erica González Apodaca (2004), "Experiencias hacia la interculturalidad de los procesos educativos. Reportes de la década de los noventa". En Ignacio Hernaiz (coord.), Educación en la diversidad. Experiencias y desafíos en la educación intercultural bilingüe. Buenos Aires: IIPE, UNESCO, pp. 19-106.

Bertely Busquets, María (2013), "Debates conceptuales sobre educación multicultural e intercultural". En María Bertely, Gunther Dietz y María Guadalupe Díaz Tepepa (coords.), Multiculturalismo y Educación 2002-2011. México: COMIE, ANUIES, pp. 41-80.

Bertely Busquets, María, Gunther Dietz y Guadalupe Díaz Tepepa (2015), "Estado de conocimiento, área 12, multiculturalismo y educación”. Presentación en el Seminario Escuela Indígenas y Etnicidad, febrero, México, CIESAS.

Caballero, Juan Julián (1989), "El maestro y el etnocidio. El caso de Huitepec, Oaxaca”. En Primeras Jornadas sobre Estudios Antropológicos Mixtecos y Mixes. Cuaderno 1. Oaxaca: CIESAS-Istmo, pp. 41-45.

Cerda García, Alejandro (2007), "Multiculturalidad y educación intercultural. Entre el neoindigenismo y la autonomía”. En Andamios, vol. 3, núm. 6, junio, pp. 97-135.

Coordinación General de Educación Intercultural y Bilingüe (2014), Programa Especial de Educación Intercultural 2014-2018. México: CGEIB-SEP.

Corona Berkin, Sarah (2008), "Políticas educativas y libros de la SEP para indígenas". En Sinéctica, núm. 3, ITESO, pp. 1-14. Disponible en: http://www. sinectica.iteso.mx/assets/files/articulos/30_politicas_ educativas_y_libros_de_la_sep_para_indigenas.pdf [consultado el 15 de febrero de 2015].

Corona Berkin, Sarah y Rebeca Pérez Daniel (2009), "Entre voces-entre culturas. La autoría dialógica hacia la participación en el espacio público". En Decisio, núm. 24, septiembre-diciembre, Centro de Cooperación Regional para la Educación de Adultos en América Latina y el Caribe, Pátzcuaro, México, pp. 15-19.

Cuevas Romo, Julio (2013), "Conocimientos locales y universales en ciencias naturales y matemáticas: reflexiones sobre su negociación desde el currículo y los materiales educativos". En Gabriel Ascencio Franco (coord.), Teoría y práctica de la educación intercultural en Chiapas. México: UNAM, pp. 213-231.

Delgado, Gabriela, Ruth Paradise, Joaquín Hernández, Adriana Robles, María Bertely y Carlos Maya (1995), "Educación indígena, de género y comunicación". En Silva Teresa Wuest (coord.), Educación, cultura y procesos sociales. México: Consejo Mexicano de Investigación Educativa, UNAM, pp. 248-310.

Díaz, E. Martín (2011), "Racismo epistémico y mono cultura: Notas sobre las diversidades ausentes en América Latina". En Revista de Epistemología y Ciencias Humanas, año 1, núm. 3, Universidad Nacional de Rosario, Universidad Nacional del Litoral, Argentina, pp.14-28. 
Dietz, Gunther (1999), “Indigenismo y educación diferencial en México: Balance de medio siglo de políticas educativas en la región Purépecha". En Revista Interamericana de Educación de Adultos, vol. 21, núm. 1, pp. 35-60.

Dietz, Gunther (2009), "Los actores indígenas ante la interculturalización de la educación superior en México: zempoderamiento o neo indigenismo?" En Revista Latinoamericana de Educación Inclusiva, vol. 3, núm. 2, pp. 55-75.

Dietz, Gunther (2014), Videoconferencia impartida el 5 de noviembre de 2014, en las instalaciones del CIESAS Pacifico Sur, en Oaxaca de Juárez, México.

Dietz, Gunther y Laura Selene Mateos Cortés (2011), Interculturalidad y educación intercultural en México. Un análisis de los discursos nacionales e internacionales en su impacto en los modelos educativos mexicanos. México: CGEIB-SEP.

Fuentes, Rocío (2010), "Convergencias y divergencias en dos discursos sobre la educación intercultural". En Cuicuilco, núm. 48, enero-julio, pp. 166-189.

González Apodaca, Erica (2013), "Cartografías de la educación intercultural en México". En Desacatos, núm. 43, pp. 201-207.

González Apodaca, Erica y Angélica Rojas Cortés (2013), "Proyectos locales, autonomía educativa y resistencia indígena". En María Bertely, Gunther Dietz y M. Guadalupe Díaz Tepepa (coords.), Multiculturalismo y educación 2002-2011. México: COMIE, ANUIES, pp. 273-293.

Haas M.,Peter (1992), "Introduction: Epistemic Communities and International Policy Coordination". En International Organization, vol. 46, núm. 1, Knowledge, Power, and International Policy Coordination, pp. 1-35.

Hale, Charles (2002), "Does Multiculturalism Menace? Governance, Cultural Rights and the Politics of Identity in Guatemala". En Journal of Latin American Studies, vol. 34, part. 3, agosto, pp. 485-524.

Hale, Charles (2004), "Racismo cultural. Notas desde Guatemala de una paradoja Americana". En Meike Hecker y Gustavo Palma (ed.), Racismoen Guatemala.De lo políticamente correcto a la lucha antirracista. Guatemala: AVANCSO, pp. 211-234.
Hale, Charles y Rosamel Millamán (2007), “iPuede el multiculturalismo ser una amenaza? Gobernanza, derechos culturales y política de la identidad en Guatemala". En María L. Lagos y Pamela Calla (coords.), Antropología del Estado. Dominación y prácticas contestatarias en América Latina. La Paz, Bolivia: INDH, PNUD, pp. 285-346.

Hamel Rainer, Enrique y Muñoz Cruz, Héctor (1981), "Bilingüismo, educación indígena y conciencia lingüística en comunidades otomíes del Valle del Mezquital en México". En Revista de Estudios Filológicos, núm. 18, pp. 127-161.

Hamel Rainer, Enrique (2000), "Políticas del lenguaje y estrategias culturales en la educación indígena". En Instituto Estatal de Educación Pública de Oaxaca (ed.), Inclusión y diversidad. Discusiones sobre la educación indígena en Oaxaca. México: IEEPO, pp. 130-167.

Hernández López, Ramón (2000), La educación para los pueblosindígenasde México. México: DGEI, Subsecretaria de Educación Básica y Normal-SEP.

Jordá Hernández, Jani (2003), Ser maestro bilingüe en Suljaá. Lengua e identidad. México: UPN, Porrúa.

Lazos Ramírez, Luz y Alejandra García Franco (2011), "La educación científica intercultural: de los beneficios teóricos a los problemas prácticos". En Revista de Derechos Humanos y Estudios Sociales, año III, núm. 6, julio-diciembre, pp.13-31.

Leyva, Xóchitl y Shannon Speed (2008), "Hacia la investigación descolonizada: nuestra experiencia de co-labor". En Xóchitl Leyva, Araceli Burguete y Shannon Speed (coords.), Gobernar (en) la diversidad: experiencias indígenas en América Latina. México: CIESAS, Facultad Latinoamericana de Ciencias Sociales, pp.34-59.

López, Luis Enrique y Wolfgang Küper (1999), "La educación intercultural bilingüe en América Latina: balance y perspectivas". En Revista Iberoamericana de Educación, mayo-agosto, núm. 20. Disponible en: http://www.rieoei.org/rie20a02.htm [consultado el 2 de marzo de 2015].

López, Luis Enrique (2006), "Desde arriba y desde abajo. Visiones contrapuestas de la educación intercultural bilingüe en América Latina”. En Christian Gros 
y Marie-Claude Strigler (eds.), Etre indien dans les Amériques: spoliations et résistance. París : Institut des Amériques, pp. 235-250.

López, Luis Enrique (2009), “Interculturalidad, educación y política en América Latina: Perspectivas desde el sur, pistas para una investigación comprometida y dialogal". En Luis Enrique López (ed.), Interculturalidad, educación y ciudadanía. Perspectivas latinoamericanas. La Paz: FUNPROEIB-Plural Editores, pp. 129-220.

Lins Ribeiro, Gustavo y Arturo Escobar (eds.) (2009), Antropologías del mundo: transformaciones disciplinarias dentro de sistemas de poder. México: The WennerGren International, CIESAS, UAM, Universidad Iberoamericana, Envión, pp. 11-42.

Maldonado Maldonado, Alma (2005a), "Comunidades epistémicas: una propuesta para estudiar el papel de los expertos en la educación Superior en México". Ponencia presentada en el VIII Congreso Nacional de Investigación Educativa, Hermosillo, Sonora. Disponible en: http://www.rdisa.org.mx/ documentos/Ponencias/Z\%20Otros\%20Eventos/ tlo_ MaldonadoMaldonado.pdf [consultado el 15 de febrero de 2015].

Maldonado Maldonado, Alma (2005b), "Comunidades epistémicas: una propuesta para estudiar el papel de los expertos en la definición de políticas en educación superior en México". En Revista de Educación Superior, vol. XXXIV (2), núm. 134, abril-junio, pp.107-122.

Medina Melgarejo, Patricia (2007), "Configuración de fronteras, interculturalidad y políticas de identidad. Niñ@s indígenas, escuela y migración (México)”. En Tramas, núm. 28, pp.17l-194.

Medina Melgarejo, Patricia (2009), "Repensar la educación intercultural en nuestras Américas". En Decisio, vol. 24, pp. 3-14.

Muñoz Cruz, Héctor (2001), "Trayectoria de las políticas de educación indígena en México”. En H. Muñoz Cruz (coord.), De prácticas y ficciones comunicativas y cognitivas en educación básica. México: UAM-Iztapalapa, pp. 433-494.
Muñoz Cruz, Héctor (2002), "Los objetivos de la educación intercultural bilingüe y sus implicaciones en la propuesta curricular de las escuelas indígenas". En Ernesto Díaz Couder (coord.), Educación intercultural bilingüe. Antología temática. México: SEP, DGEI, pp. 9-26

Muñoz Cruz, Héctor (2008), "Indígenas y educación intercultural en América Latina”. En A. Cura de Gianna y Carla Marres (eds.), Marginalitá ed egemoniain America Latina e Mediterraneo. Roma: Presso Arti Grafiche La Moderna.

Olivé, León (2009), "Por una auténtica interculturalidad basada en el reconocimiento de la pluralidad epistemológica". En L. Tapia (ed.), Pluralismo epistemológico. La Paz, Bolivia: Muela del Diablo, Comuna, CLACSO, CIDES-UMSA, pp. 19-30.

Pérez Pérez, Elías (2003), La crisis de la educación indígena en el área tzotzil. Los Altos de Chiapas. México: Porrúa, UPN.

Pérez Ruiz, Maya Lorena (2009), “iDe qué hablamos cuando nos referimos a lo intercultural? Reflexiones sobre su origen, contenidos, aportaciones y limitaciones". En Laura Valladares, Maya L. Pérez Ruiz y Margarita Zárate Vidal (coords.), Estados plurales. El reto de la diversidad y la diferencia. México: UAM Ixtapalapa, pp. 199-228.

Pineda, Luz Olivia (1993), Caciques culturales: el caso de los maestros bilingües en los Altos de Chiapas. México: Altres Costa-Amic.

Ramírez Castañeda, Elisa (2006), La educación indígena en México. México: PUMC, UNAM.

Rockwell, Elsie y Erica González Apodaca (2012), "Anthropological Research on Educational Processes in México". En Kathryn Anderson-Levitt (ed), Mapping Anthropologies of Education. A global Guide to Ethnographic Studies of Learning and Schooling. Oxford, Nueva York: Bergham Books.

Schmelkes, Sylvia (2004), "La educación intercultural: un campo en proceso de consolidación". En Revista Mexicana de Investigación Educativa, vol. 9, núm. 20, pp. 9-13.

Vallejo Ruiz, Mónica, Almudena Ocaña Fernández, Ángel Bueno Sánchez, Manuel Torralbo Rodríguez 
y Antonio Fernández Cano (2005), "Producción científica sobre educación multicultural contenida en las bases de datos Social Sciences Citation Index y Arts G Humanities Citation Index (1956-2003)". En Revista Especializada de Docencia Científica, núm. 28, vol. 2, pp. 206-220.
Vargas, María Eugenia (1994), Educación e ideología: constitución de una categoría de intermediarios en la comunicación interétnica: el caso de los maestros bilingües tarascos (1964-1982). México: CIESAS.

Villoro, Luis (2006), Creer, saber, conocer. México: Siglo XXI. 